\title{
0018 SAFE BABY UNIVERSITY
}

KRosenthal* ${ }^{*}$ S J Smith, J Moss, R Schwenn, RDavis, LCain-Martin Correspondence: Children's Hospital of Michigan, 3901 Beaubien, Detroit, MI 48201, USA

\subsection{6/ip.2010.029215.18}

Purpose Unsafe sleep is the number one killer of children from 1 month to 1 year of age. In Wayne County, infants are at high risk for death due to unsafe sleep. Detroit has the highest infant mortality in Michigan.

Methods Safe Baby university addresses the lack of knowledge that parents/caregivers have regarding unsafe sleep and other top causes of injury and death for infants, including child passenger safety, nutrition, fire/burn and preventing shaken baby. Participants learn safe sleep practices including risk factors for unsafe sleep, the correct installation/proper use of child restraints, how to mix formula correctly, discussions about what to do when your baby will not stop crying and how to assess home for fire and burn hazards. Skill stations are used for teaching. Additionally, if their home needs smoke/carbon monoxide alarms, families sign-up to have them installed free of charge (CDC SAIFE grant). At the conclusion of the class, families receive one free car seat or pack'n play.

Results The pretest is composed of 18 questions; seven personal information questions, six questions based on the injury prevention topics, with five specifically related to safe sleep. The post-test is comprised of 19 questions. The pretest average score was $77 \%$. The post test average score was $90.9 \%$. Showing an overall increase of $18 \%$. The increase in post-test score is statistically significant using a CI of $95 \%$ we obtained z-score of 2.29 . 